\title{
The impact of oxidative DNA changes and ATM expression on morphological and functional activities on hepatocytes obtained from mesenchymal stem cells
}

\author{
Shahnaz Esmaeli ${ }^{1}$, Abdolamir Allameh ${ }^{1}$, Maryam Adelipour ${ }^{1}$, Masoud Soleimani ${ }^{2}$ and Fatemeh \\ Rahbarizadeh $^{3}$ \\ ${ }^{1}$ Department of Clinical Biochemistry, Faculty of Medical Sciences, Tarbiat Modares University, Tehran, Iran \\ ${ }^{2}$ Department of Hematology, Faculty of Medical Sciences, Tarbiat Modares University, Tehran, Iran \\ ${ }^{3}$ Department of Medical Biotechnology, Faculty of Medical Sciences, Tarbiat Modares University, Tehran, Iran \\ *Corresponding author \\ Department of Biochemistry, Faculty of Medical Sciences, Tarbiat Modares University, POB 14115-111, Tehran, \\ Iran. E-mail: Allameha@modares.ac.ir
}

\begin{abstract}
Resistance to oxidative damages in undifferentiated mesenchymal stem cells (MSCs) in comparison with the undifferentiated progenitor cells may differ depending on several factors. This study was carried out to examine the impact of hepatogenic differentiation process of MSCs on oxidative DNA damage markers. Hepatic differentiation of MSCs was carried out using a two-step conventional protocol and the cells were processed for characterization using morphological and biochemical markers. During the course of differentiation cellular levels of reactive oxygen species (ROS), 8-hydroxy-2'-deoxyguanosine (8-OH-dG) and expression of ataxia-telangiectasia mutated (ATM) protein were estimated at time intervals (10, 20 and 30 days). The results showed a decrease in cellular ROS $(13 \%, \mathrm{P}<0.05)$ at early stages of hepatogenic differentiation. Similarly, there was a small but significant decrease in 8-OH-dG level and ATM expression in differentiated hepatoctytes. Despite the small changes in oxidative damage factors and ATM expression during the differentiation process, the hepatocytes obtained were morphologically and biologically intact.
\end{abstract}

Keywords: ATM; Differentiation; Hepatocyte-like cells; 8-OH-dG; ROS. 


\section{Introduction}

Proliferation and differentiation of stem cells are two important physiological events that are associated with various molecular and biochemical factors. Umbilical cord (UC) mesenchymal stem cells (MSCs) are well known sources of multipotent stem cells which can differentiate successfully to hepatocyte-like cells [1] and are candidate for stem cell therapy particularly in hepatic diseases such as liver fibrosis [2]. The changes which occur during differentiation of the stem cells rely on the selective media and stimuli applied in the differentiation processes.

Earlier we reported that when MSCs from human bone marrow are differentiated to hepatocytelike cells parallel to the liver specific markers such as albumin, the expression of enzymes involved in drug metabolism also tend to increase [3]. In this connection we also reported that the extent of DNA damage induced by aflatoxin B1 (a potent hepatocarcinogen) in MSCs and hepatocyte-like cells differs which is associated with the changes in cytochrome P450 3A4 (CYP3A4) expression in cells before and after differentiation [4]. More recently we reported that during differentiation of MSCs isolated from human umbilical cord blood (UCB) to hepatocytelike cells there is an increasing pattern of expression of cyclooxygenase-2 (COX-2) which was associated with increased protein and lipid oxidation [5].

The genomic integrity and prevention of oxidative DNA damage during the stem cell differentiation are important issues which should be taken into consideration when the cells are used for preclinical and clinical applications. A certain amount of endogenous Reactive oxygen species (ROS) is generated in the cells as a result of normal cellular metabolism. Also, ROS are generated under pathologic processes such as inflammation, or in response to growth factors and cytokines [6, 7].Depending on their cellular concentration, ROS can interact with cellular biomolecules such as DNA, modulate the activity of the proteins and genes responding to stress and regulate the genes which are related to cell proliferation and differentiation [8, 9]. Their effect on the differentiation process depends on stem cell origin and protocol used in the differentiation [10].

Among the markers of oxidative DNA damage, 8-OH-dG is the most commonly produced base lesion, and measured as an index of oxidative DNA damage [11]. 8-OH-dG is strongly 
mutagenic and leads to $\mathrm{G}: \mathrm{C}$ to $\mathrm{T}: \mathrm{A}$ transversion mutations capable of carcinogenic initiation [12].

One of the most important proteins involved in sensing the redox status is the ataxiatelangiectasia mutated protein (ATM) [13]. This protein is a member of the phosphatidylinositol 3-kinaselike family of serine/threonine protein kinases (PIKKs) [14], which activates cell-cycle checkpoints and DNA repair proteins [15] and controls mitochondrial respiratory rate and functions [16]. ATM is activated in response to increased levels of oxidative stress caused by ROS [17] and protects the cell from oxidative damage by stimulating antioxidant defenses in the host cells [18]. Moreover, the role of ATM in the maintenance, proliferation, differentiation, and survival of stem cells was demonstrated [19, 20].

Recently we reported that the hepatogenic differentiation of stem cells is associated with the changes in cellular fatty acid profile and PPAR- $\alpha$ expression. The maturation stage of the cells was independent of ROS production and lipid peroxidation in developing hepatocytes-like cells suggesting that hepatocyte formation from stem cells is associated with a shift in the fatty acid profile as normal phenomenon in hepatogenic differentiation of the MSCs [21]. The present study is aimed to examine the relationship between hepatogenic differentiation on oxidative DNA damage and MSC differentiation to biologically active hepatocyte-like cells. Moreover the changes in ATM expression in presence of hepatic differentiation stimuli have been studied.

\section{Materials and methods}

\section{Umbilical cord tissues and isolation of mesenchymal stem cells (UC-MSC)}

Human umbilical cord samples were obtained from three full-term infants with the informed consent of the mothers (Samples were received from Milad Hospital, Tehran, Iran).The cords were rinsed twice by phosphate-buffered saline (PBS) (Invitrogen-Gibco, USA) containing penicillin and streptomycin (Invitrogen-Gibco, USA). After removal of blood vessels, the cords were cut into small pieces $\left(\sim 2 \mathrm{~mm}^{3}\right)$ with a sterile scalpel, placed into 6 well plates and floated in low-glucose Dulbecco’s Modified Eagle’s Medium (DMEM-LG) (Invitrogen-Gibco, USA) containing12\% fetal bovine serum (FBS) (Invitrogen-Gibco, USA), penicillin and streptomycin. The pieces of cord were subsequently incubated at $37^{\circ} \mathrm{C}$ with $5 \% \mathrm{CO} 2$. After 3 weeks tissue pieces were removed. Adherent cells were passaged upon reaching $70 \%$ confluence. 
Characterization of the MSCs was routinely examined by flow cytometry analysis as described in our recent publication [21].

\section{Hepatogenic differentiation of the MSCs to hepatocyte-like cells}

At the P3 passage, the MSCs $\left(2 \times 10^{3}\right.$ cells $\left./ \mathrm{cm}^{2}\right)$ were seeded into $0.1 \%$ gelatin-coated wells in a 24-well plate. Hepatic differentiation was carried out using a two-step protocol: step-one differentiation medium, consisting of DMEM containing 5\% FBS, 20 ng/ml Hepatocyte growth factor (HGF) (ProSpec, USA), and $10 \mathrm{ng} / \mathrm{ml}$ fibroblast growth factor-4 (FGF-4) (Millipore, USA) for 2 weeks followed by treatment with step-two maturation medium, consisting of DMEM supplemented with 5\% FBS, 20 ng/ml HGF, 20 ng/ml oncostatin-M (OSM) (Genscript, USA), $10^{-6} \mathrm{M}$ dexamethasone (DEX) (Sigma-Aldrich, USA). Media change was performed twice weekly. During this period the formation of hepatocyte-like cells were monitored by detecting different liver-specific markers as follows:

\section{Expression of liver specific markers in hepatocyte-like cells}

The expression of liver-specific markers was assayed in cells using reverse transcription polymerase chain reaction (RT-PCR). Briefly, total RNA was extracted from the MSCs and hepatocyte-like cells recovered at different time intervals during differentiation. Likewise RNA was extracted from HepG2 established cell line and considered as positive control. RNA was extracted using the Nucleo Spin ${ }^{\circledR}$ RNA II kit (Macherey-Nagel, Germany), and the synthesis of cDNA was performed using $0.5 \mu \mathrm{g}$ RNA with First Strand cDNA Synthesis Kit (Fermentas, Canada), according to the manufacturer's instruction. PCR amplification was performed using PCR master mix (Fermentase, Canada) with denaturation at $95{ }^{\circ} \mathrm{C}$ for $15 \mathrm{~s}$, annealing temperature for $30 \mathrm{~s}$ depending on the primers, extension at $72{ }^{\circ} \mathrm{C}$ for $60 \mathrm{~s}$. The primers and product lengths are listed in Table 1.

\section{Detection of albumin in the cells by immunofluorescence technique}

To assess the expression of albumin in hepatocyte-like cells and their progenitors, the cells were fixed with 4\% paraformaldehyde for 20 min (Sigma-Aldrich, USA), permeabilized with 0.25\% Triton X-100 (Sigma-Aldrich, USA), blocked for $60 \mathrm{~min}$ at room temperature with 5\% bovine serum albumin (BSA) (Sigma-Aldrich, USA), and incubated for overnight at $4{ }^{\circ} \mathrm{C}$ with mouse monoclonal anti-albumin (Minneapolis, USA) (1: 250). The cells were washed 3 times with 
PBS-Tween-20 (PBST, 0.1\%) and incubated with fluorescence labeled secondary antibody (FITC-labeled goat anti-mouse IgG, dilution 1:100) for $60 \mathrm{~min}$ at room temperature. After rinsing with PBS, the nuclei were counterstained with 4', 6-diamidino-2-phenylindole (DAPI) (Sigma-Aldrich, USA), and the cells were observed under the fluorescent microscope (Olympus, DP72, Japan).

\section{Determination of glycogen storage using periodic acid-schiff (PAS) staining}

Culture dishes containing hepatocyte-like cells on 30 day of the differentiation and the MSCs were fixed in $4 \%$ paraformaldehyde for $15 \mathrm{~min}$ at room temperature. The fixed cells were oxidized in 1\% periodic acid for 5 min and rinsed 3 times in deionized water. The cells were then treated with Schiff's reagent for $15 \mathrm{~min}$, and rinsed in deionized water for 5-10 min. Samples were assessed using microscope (Olympus, DP72, Japan).

\section{Estimation of cellular ROS}

To measure ROS generation during hepatogenic differentiation, the MSCs before and after differentiation to hepatocyte-like cells were incubated with $10 \mu \mathrm{M}$ of 2', 7'-dichloroflurescein diacetate (DCF-DA) (Marker Gene Technology (MGT), Italy) for $30 \mathrm{~min}$. The reaction was performed in dark to avoid the degradation of DCF-DA. Then, the cells were harvested, washed once, and the cell pellet resuspended in PBS. The fluorescence intensity was monitored at wavelengths of $480 \mathrm{~nm}$ excitation and $525 \mathrm{~nm}$ emission settings on a spectrofluorometer (BioTek, USA).

\section{Changes in 8-OH-dG during hepatogenic differentiation of MSCs}

In this study 8-OH-dG is used as a biomarker of DNA damage in MSCS and hepatocyte-like cells during differentiation. For this purpose, DNA was extracted from the cell samples using a DNA extraction kit (Qiagen, USA). The assay was carried out with $3 \mu$ g of DNA sample and quantitative estimation of 8-OH-dG was performed using a HT 8-OH-dG ELISA Kit II according to the manufacturer's instruction (Trevigen.Inc, USA). The colorimetric substrate was monitored at wavelengths $450 \mathrm{~nm}$ on SUNRISE absorbance reader (TECAN, Austria).

\section{Comparison of ATM expression during hepatogenic differentiation of MSCs}

ATM expression at mRNA levels was measured by quantitative PCR. Briefly, total RNA and cDNA were synthesized as described in RT-PCR section. Quantitative PCR was performed by 
using a Rotor Gene system (Corbett Research, Australia) and SYBR Green detection (iQ ${ }^{\mathrm{TM}}$ SYBR ${ }^{\circledR}$ Green Supermix, Bio-Rad, USA). Primers were designed using Allele ID software, and further reanalyzed with the Oligo software program. The following primers were used in Q-PCR: ATM (F), 5'-AGCAAAGAAGTAGAAGGAACCAG-3' and ATM (R), 5'GGGATAGAGCGAATACACAGAC-3' (product size: 168 bp, Gene bank code: NM-000051.3); for GAPDH (F), 5'-GAGTCCACTGGCGTCTTCACC-3' and GAPDH (R), 5'AGGCATTGCTGATGATCTTGAGG-3' (product size: 163 bp, Gene bank code: NM002046.3). GAPDH as a housekeeping gene was used to normalize the mRNA expression levels, and the following program was used: step1 initial denaturation: $95^{\circ} \mathrm{C}$ for $3 \mathrm{~min}$, step2: $95^{\circ} \mathrm{C}$ for $15 \mathrm{~s}$, annealing temperature at $60{ }^{\circ} \mathrm{C}$ for $30 \mathrm{~s}$, and extension at $72{ }^{\circ} \mathrm{C}$ for $60 \mathrm{~s}$. Serial dilutions of the cDNA samples were used to construct the standard curve and for determining the real-time PCR efficiency for each primer pair.

\section{Localization of ATM in the cells by immunocytochemistry (ICC) analysis}

ATM and phosphorylated ATM expression was estimated using ICC technique in differentiated and undifferentiated cells maintained in 4-well plates. For this purpose, the cells were rinsed twice with PBS and ICC was performed as described in the 'Methods section'. The antibodies used for ICC analysis of ATM were: Rabbit anti-human ATM (1:200), Rabbit anti-human ATM (phospho S1981) (1:200) and fluorescence labeled secondary antibody (FITC-labeled Goat antiRabbit IgG at 1:1000) (Abcam, UK). After rinsing with PBS, the nuclei were counterstained with DAPI, and the cells were observed under the fluorescent microscope (Olympus, DP72, Japan).

\section{Statistical Analysis}

All the statistical analyses were performed using the SPSS statistic version 17 software. Data were analyzed using one-way ANOVA in cell samples collected at time intervals during hepatocyte differentiation. All experiments were carried out in triplicate and the data are presented as mean \pm standard deviation (SD). $\mathrm{P}$ value $<0.05$ is considered as significant.

\section{Results}

\section{Functional characterization of hepatocyte-like cells}


During the hepatic differentiation, the cells exhibited an apparent transition from fibroblast-like morphology to round shape and a cubical morphology (Fig. 1). The expression of selected hepatocyte-specific markers was measured as a part of characterization of hepatogenic differentiation. Expression of albumin (ALB), and cytokeratin 18 (CK-18) at mRNA levels were carried out by RT-PCR. ALB was expressed in undifferentiated cells at low levels, but its expression was significantly up regulated after differentiation. CK-18 gene was expressed in undifferentiated and differentiated cells. $\mathrm{TDO}_{2}$ gene was expressed on day 30 of differentiation (Fig. 2).

Based on ICC data, albumin was not detectable in MSCs before differentiation; however, hepatocytes collected on day 30 of differentiation showed albumin accumulation in the cells (Fig. 3). Likewise glycogen granules were not detectable in the MSCs, but positively stained glycogen granules were detected in the cytoplasm of differentiated MSCs on day 30 of differentiation (Fig. 4).

\section{Changes in cellular ROS during differentiation}

The intracellular ROS production during differentiation was assayed following incubation of the cells with $10 \mu \mathrm{M}$ DCF-DA. After deacetylation, DCF quantitatively reacts with ROS (mainly $\mathrm{H}_{2} \mathrm{O}_{2}$ ) to produce the fluorescent dye DCF, which remains trapped within the cell. The results showed that ROS generation in hepatocyte-like cells on day 10 of differentiation was significantly $(\mathrm{P}<0.05)$ lower than undifferentiated cells. However, in case of cell collected on day 30, reached to the levels measured in undifferentiated MSCs (Fig. 5).

\section{Oxidative DNA damage in MSCs and hepatocyte-like cells}

The 8-OH-dG was measured in the cells as an indicator of oxidative DNA damage. The assay was performed on the DNA samples extracted from the MSCs before differentiation and hepatocyte-like cells during the course of differentiation. As shown in figure 6, the level of 8$\mathrm{OH}-\mathrm{dG}$ was relatively higher in undifferentiated cells. Whereas; there was a decline in the 8-OH$\mathrm{dG}$ generation during the differentiation, this decrease was statistically significant $(21 \% ; \mathrm{P}<0.05)$ in hepatocyte-like cells recovered on day 30 of differentiation.

\section{Expression of ATM in MSCs during differentiation into hepatocyte-like cells}


The Q-PCR data presented in figure 7, compares the ATM expression at mRNA levels in differentiated and undifferentiated cells during hepatogenic differentiation. The expression of ATM specific mRNA in undifferentiated MSCs was significantly higher $(\mathrm{P}<0.05)$ compared to the differentiated cells. The difference in ATM expression was statistically significant between the undifferentiated and the differentiated hepatocytes collected on days 10, 20 and 30 of differentiation.

\section{Localization of ATM protein in MSCs before and after hepatic differentiation}

Based on ICC data it was demonstrated that in undifferentiated MSCs and hepatocyte-like cells the ATM protein is mainly accumulated in the nuclear regions of the cells. There were no noticeably differences in ATM protein accumulation in hepatocyte-like cells and their progenitor MSCs (Fig. 8). However, the accumulation of the phosphorylated ATM (phospho S1981) in the cells was decreased in hepatocyte-like cells following hepatic differentiation. In this case, the accumulation of phosphorylated ATM was not limited to nuclear regions, and was expressed at high levels in both cytoplasmic and nuclear regions in undifferentiated MSCs; but, following differentiation its expression is confined to nuclear regions of the hepatocyte-like cells (Fig. 9).

\section{Discussion}

Differentiation of MSCs into hepatocytes-like cells in culture media under normal condition can be associated with metabolic changes leading to oxidative damage. ROS is a key factor in balancing the metabolic signaling and cellular damage during differentiation. Differentiationrelated changes in ROS concentration can cause oxidative DNA damage and induction of DNA repair intermediates[22, 23].

The mutagenic lesion by 8-Oxo-Gua as a marker of DNA damage occurs either spontaneously or as result of exogenous factors[11]. During the differentiation process of a stem cell, depending on the stimuli and signals the expression and regulation of genes requires for a specialized cell function will be activated. For instance differentiation of MSCs into hepatocyte-like cells is associated with increased expression of liver-specific genes such as, albumin, alpha-fetoprotein and CYP3A4 [24, 25]. During this process there are possibilities of changes in ROS production and oxidative damage levels of macromolecules, particularly DNA. The hepatogenic 
differentiation of the stem cells is associated with developmental changes in liver specific markers such as CYP450s and enzymes involved in glutathione (GSH) conjugation pathway [3].

Cells are constantly exposed to ROS and they possess the defense mechanism against ROS production [26]. It appears that the differentiation process induced by HGF is responsible for changes in ROS production, 8-OH-dG levels and ATM expression. However, under these circumstances, the development of hepatocyte-like cells and functions are normal as shown by liver function parameters.

The part played by the HGF in modulation of ROS production cannot be ruled out [27]. Studies on the role of ROS in differentiation of stem cells from different sources used for differentiation into mesendodermal lineage [10] or osteoblasts [23] show that ROS at low-to-moderate concentrations can act as signaling molecule and play a role in signaling pathways in cellular biology [8].

Expression of albumin during hepatogenic differentiation along with morphological changes of the cells indicate the slowdown of the proliferation rate of the stem cells following initiation of hepatogenic differentiation. Decrease in ROS production and related factors in hepatocytes at early stage of differentiation can be assigned to a decrease in the cell population as shown by Brdu assay (Result not shown).

In the present study, it was shown that in hepatogenic differentiation of MSCs carried out in absence of any mutagenic agent there is a decline in the level of 8-OH-dG in hepatocytes compared to undifferentiated MSCs (Fig. 6). A decrease in 8-OH-dG in developing hepatocytelike cells on day 30 of differentiation was associated with down regulation of ATM, indicating the part played by ATM in regulation of DNA damage-repair system as well as cell proliferation. The down regulation of ATM during stem cell differentiation is justified by its role in maintenance, self-renewal, and differentiation of certain stem cells [19, 20, 28].

Changes in the 8-OH-dG formation were associated with a decrease in cytoplasmic accumulation of the phosphorylated ATM in the cytoplasm cells compared to undifferentiated MSCs (Fig. 7). In case of MSCs having relatively high proliferation capacity, ATM may be required to eliminate cellular damages before they have time to divide to form "abnormal” progenitor cells. Hence, a decrease in the ATM expression in the differentiated cells is considered as a part of the DNA 
damage-repair system which is changes depending on the cell status. Expression and activity of ATM differ depending on cell type and cell cycle phase [29]. This information was further supported by the results of Lu et al., (2005) showing that the ATM protein levels is associated with increased DNA synthesis during liver regeneration and ATM deficiency impaired the ability of hepatocytes to proliferate. The proliferation capacity and the density of the hepatocyte-like cells in culture may be considered as a reason for decreased ATM expression. This was evidenced by showing that cell growth at higher cell density in cultures decrease their proliferation and metabolic activity [30] and ATM expression levels were reduced in cells maintained at higher densities [31]. In this line it has been reported that the redox state of the cell can be affected by ATM expression in a direct or indirect manner and ATM can sense and respond to increased ROS levels [32]. Recently, Alexander et al., (2010) identified a cytoplasmic pathway for ROS-induced ATM activation of TSC2 to regulate mTORC1 signaling and autophagy, identifying an integration node for the cellular damage response with key pathways involved in metabolism, protein synthesis, and cell survival.

Although, it is unclear how ATM is linked to oxidative stress management during hepatogenic differentiation, its expression in the MSCs before and after differentiation indicates that ATM plays an important role in the control of cellular redox status and genome maintenance during hepatic differentiation especially in the MSCs to cope with probable genotoxic stress caused by ROS. The changes in ATM and the 8-OH-dG in the cells differentiated in absence of a mutagenic agent may not be implicated in the cells treated with a mutagen. Perhaps, hepatogenic differentiation of MSCs carried out in presence of aflatoxin B1 (a potent hepatocarcinogen) showed that the DNA damages were accumulated in hepatocytes-like cells and the damages were significantly higher compared to their undifferentiated progenitor cells. The extensive DNA damage measured by Comet assay in hepatocytes-like cells was assigned to the over expression of cytohrome P-450 (CYP3A4) which was increased during hepatogenic differentiation of MSCs [4].

A small decrease in 8-OH-dG in developing hepatocyte-like cells on day 30 of differentiation was associated with down regulation of ATM, indicating the part played by ATM in regulation of DNA damage-repair system as well as cell proliferation. The decline in 8-OH-dG was associated with lower accumulation of the phosphorylated ATM in the cytoplasm of hepatocytes- 
like cells compared to undifferentiated MSCs suggesting that ATM may play an important role in the control of cellular redox status and genome maintenance during hepatic differentiation especially in the MSCs to cope with probable genotoxic stress caused by ROS. The ubiquitous expression of ATM in the MSCs was consistent with the role for ATM in genome maintenance during cell division at the site where cells are undergoing rapid proliferation [33]. The attenuation of ATM expression following hepatic differentiation in the differentiated cells on one hand can protect the cells against apoptosis caused by ATM signaling pathway, on the other hand increases cellular susceptibility to DNA damage. In this line earlier we showed that hepatocytelike cells derived from human umbilical cord blood MSCs are vulnerable than their progenitors into cellular damage caused by aflatoxin B1 [4].

It has been showed that growth factors such as HGF can decrease the rate of MSCs proliferation [34]. Moreover, initial plating density can affect the genes regulating cell proliferation [35]. Furthermore, it has been reported that during liver regeneration, ATM protein levels is associated with increased DNA synthesis and the ability of hepatocytes to proliferate is impared in case of ATM deficiency [36].

Moreover, ATM expression levels were reduced in cells maintained at higher densities [31]; therefore, the reduction of ATM expression and 8-OH-dG levels observed during hepatic differentiation might be related to a decline in cellular proliferation capacity of differentiated cells, or as result of increased cellular density in culture media which needs further investigation.

Based on the data presented in this paper, it appears that although there was a small changes in cellular ROS, DNA adduct (8-OH-dG) and ATM expression, but the process of hepatogenic differentiation of MSCs was accomplished normally. The hepatocytes-like cells were proved to morphologically and biologically normal.

\section{Acknowledgments}

This study was supported by a grant (project \# 90007389) provided by Iran National Science Foundation (INSF).

\section{Conflict of interest}

The authors declare that no competing financial interests exist. 


\section{References}

[1] Zhang Y-N, Lie P-C, Wei X. Differentiation of mesenchymal stromal cells derived from umbilical cord Wharton's jelly into hepatocyte-like cells. Cytotherapy. 2009;11:548-58.

[2] Tsai PC, Fu TW, Chen YMA, Ko TL, Chen TH, Shih YH, et al. The therapeutic potential of human umbilical mesenchymal stem cells from Wharton's jelly in the treatment of rat liver fibrosis. Liver Transplantation. 2009;15:484-95.

[3] Allameh A, Esmaeli S, Kazemnejad S, Soleimani M. Differential expression of glutathione Stransferases P1-1 and A1-1 at protein and mRNA levels in hepatocytes derived from human bone marrow mesenchymal stem cells. Toxicology in Vitro. 2009;23:674-9.

[4] Ghaderi M, Allameh A, Soleimani M, Rastegar H, Ahmadi-Ashtiani HR. A comparison of DNA damage induced by aflatoxin B1 in hepatocyte-like cells, their progenitor mesenchymal stem cells and CD34+ cells isolated from umbilical cord blood. Mutation Research/Genetic Toxicology and Environmental Mutagenesis. 2011;719:14-20.

[5] Khajeniazi S, Allameh A, Soleimani M, Mortaz E. Changes in COX-2 and oxidative damage factors during differentiation of human mesenchymal stem cells to hepatocyte-like cells is associated with downregulation of p53 gene. Biological chemistry. 2013;394:1213-22.

[6] Thannickal VJ, Fanburg BL. Reactive oxygen species in cell signaling. American Journal of PhysiologyLung Cellular and Molecular Physiology. 2000;279:L1005-L28.

[7] Williams GM, Jeffrey AM. Oxidative DNA damage: endogenous and chemically induced. Regulatory Toxicology and Pharmacology. 2000;32:283-92.

[8] Valko M, Leibfritz D, Moncol J, Cronin MT, Mazur M, Telser J. Free radicals and antioxidants in normal physiological functions and human disease. The international journal of biochemistry \& cell biology. 2007;39:44-84.

[9] Schreck R, Albermann K, Baeuerle PA. Nuclear factor kB: an oxidative stress-responsive transcription factor of eukaryotic cells (a review). Free radical research communications. 1992;17:221-37.

[10] Ji A-R, Ku S-Y, Cho MS, Kim YY, Kim YJ, Oh SK, et al. Reactive oxygen species enhance differentiation of human embryonic stem cells into mesendodermal lineage. Experimental \& molecular medicine. 2010;42:175-86.

[11] Novo E, Parola M. Redox mechanisms in hepatic chronic wound healing and fibrogenesis. Fibrogenesis \& tissue repair. 2008;1:5.

[12] Halliwell B. Oxidative stress and cancer: have we moved forward? Biochem j. 2007;401:1-11.

[13] Sasaki M, Ikeda H, Sato Y, Nakanuma Y. Proinflammatory cytokine-induced cellular senescence of biliary epithelial cells is mediated via oxidative stress and activation of ATM pathway: a culture study. Free radical research. 2008;42:625-32.

[14] Shiloh Y. ATM and ATR: networking cellular responses to DNA damage. Current opinion in genetics \& development. 2001;11:71-7.

[15] Kastan MB, Lim D-s. The many substrates and functions of ATM. Nature Reviews Molecular Cell Biology. 2000;1:179-86.

[16] Ambrose M, Goldstine JV, Gatti RA. Intrinsic mitochondrial dysfunction in ATM-deficient lymphoblastoid cells. Human molecular genetics. 2007;16:2154-64.

[17] Alexander A, Walker CL. Differential localization of ATM is correlated with activation of distinct downstream signaling pathways. Cell Cycle. 2010;9:3709-10.

[18] Jung M, Kondratyev A, Lee SA, Dimtchev A, Dritschilo A. ATM gene product phosphorylates IKB- $\alpha$. Cancer research. 1997;57:24-7.

[19] Allen DM, van Praag H, Ray J, Weaver Z, Winrow CJ, Carter TA, et al. Ataxia telangiectasia mutated is essential during adult neurogenesis. Genes \& development. 2001;15:554-66. 
[20] Ito K, Hirao A, Arai F, Matsuoka S, Takubo K, Hamaguchi I, et al. Regulation of oxidative stress by ATM is required for self-renewal of haematopoietic stem cells. Nature. 2004;431:997-1002.

[21] Esmaeli S, Allameh A, Soleimani M, Rahbarizadeh F, Frouzandeh-Moghadam M. The role of albumin and PPAR- $\alpha$ in differentiation-dependent change of fatty acid profile during differentiation of mesenchymal stem cells to hepatocyte-like cells. Cell biochemistry and function. 2014;32:410-9.

[22] Sauer H, Wartenberg M, Hescheler J. Reactive oxygen species as intracellular messengers during cell growth and differentiation. Cellular Physiology and Biochemistry. 2001;11:173-86.

[23] Chen CT, Shih YRV, Kuo TK, Lee OK, Wei YH. Coordinated changes of mitochondrial biogenesis and antioxidant enzymes during osteogenic differentiation of human mesenchymal stem cells. Stem Cells. 2008;26:960-8.

[24] Lee H-J, Jung J, Cho KJ, Lee CK, Hwang S-G, Kim GJ. Comparison of in vitro hepatogenic differentiation potential between various placenta-derived stem cells and other adult stem cells as an alternative source of functional hepatocytes. Differentiation. 2012;84:223-31.

[25] Kazemnejad S, Allameh A, Soleimani M, Gharehbaghian A, Mohammadi Y, Amirizadeh N, et al. Biochemical and molecular characterization of hepatocyte-like cells derived from human bone marrow mesenchymal stem cells on a novel three-dimensional biocompatible nanofibrous scaffold. Journal of gastroenterology and hepatology. 2009;24:278-87.

[26] Hirano T. Differentiation of Embryonic Stem Cells and Oxidative DNA Damage/DNA Repair Systems. Journal of Stem Cell Research \& Therapy. 2012.

[27] Ozaki M, Haga S, Zhang H, Irani K, Suzuki S. Inhibition of hypoxia/reoxygenation-induced oxidative stress in HGF-stimulated antiapoptotic signaling: role of PI3-K and Akt kinase upon rac1. Cell Death \& Differentiation. 2003;10:508-15.

[28] Sherman $\mathrm{MH}$, Bassing $\mathrm{CH}$, Teitell MA. Regulation of cell differentiation by the DNA damage response. Trends in cell biology. 2011;21:312-9.

[29] Bartkova J, Bakkenist CJ, Meyts ER-D, Skakkebæk NE, Sehested M, Lukas J, et al. ATM activation in normal human tissues and testicular cancer. Cell Cycle. 2005;4:838-45.

[30] Darzynkiewicz Z, Traganos F, Staiano-Coico L, Kapuscinski J, Melamed M. Interactions of rhodamine 123 with living cells studied by flow cytometry. Cancer research. 1982;42:799-806.

[31] Tanaka T, Halicka HD, Huang X, Traganos F, Darzynkiewicz Z. Constitutive histone H2AX phosphorylation and ATM activation, the reporters of DNA damage by endogenous oxidants. Cell cycle. 2006;5:1940-5.

[32] Barzilai A, Rotman G, Shiloh Y. ATM deficiency and oxidative stress: a new dimension of defective response to DNA damage. DNA repair. 2002;1:3-25.

[33] Oricchio E, Saladino C, lacovelli S, Soddu S, Cundari E. ATM is activated by default in mitosis, localizes at centrosomes and monitors mitotic spindle integrity. Cell cycle. 2006;5:88-92.

[34] Neuss S, Becher E, Wöltje M, Tietze L, Jahnen-Dechent W. Functional expression of HGF and HGF receptor/c-met in adult human mesenchymal stem cells suggests a role in cell mobilization, tissue repair, and wound healing. Stem cells. 2004;22:405-14.

[35] Lee MW, Kim DS, Ryu S, Jang IK, Kim HJ, Yang JM, et al. Effect of ex vivo culture conditions on immunosuppression by human mesenchymal stem cells. BioMed research international. 2013;2013. [36] Lu S, Shen KC, Wang Y, Brooks S, Wang YA. Impaired hepatocyte survival and liver regeneration in Atm-deficient mice. Human molecular genetics. 2005;14:3019-25.

Figures legend 
Figure 1. Morphological changes of mesenchymal stem cells (MSCs) during differentiation to hepatocytes-like cells.

The undifferentiated MSCs (A) and hepatocyte-like cells on day 10 (B), day 20 (C), and day 30 of differentiation (D). Scale bars represent $50 \mu \mathrm{m}$. The arrows indicate differentiated cells with double nuclei in the later stage of differentiation.

Figure 2. Expression of liver specific markers at mRNA levels in the mesenchymal stem cells before and after hepatic differentiation.

Expression of albumin (ALB), cytokeratin 18 (CK-18), and tryptophan2, 3-dioxygenase (TDO2) were estimated during hepatogenic differentiation in the undifferentiated cells (lane 1), differentiated cells on day 30 (lane 2) of differentiation. The HepG2 cell line (lane 3) was used as positive control.

Figure 3 .Immunocytochemical analysis of albumin protein in mesenchymal stem cells and hepatocyte-like cells during hepatic differentiation.

Albumin expression in hepatocyte-like cells on 30 day after differentiation (B), undifferentiated cells (C), and in HepG2 cells as positive control cells (A).

Figure 4. Glycogen storage determined by periodic acid schiff(PAS) staining in hepatocyte-like cells and their progenitors.

Positively stained glycogen granules (A) were detected in the cytoplasm of differentiated MSCs after 4 weeks of differentiation. Undifferentiated MSCs was negative for periodic acid schiff (PAS) staining (B).

Figure 5.Intracellular reactive oxygen species (ROS) formation during hepatic differentiation of mesenchymal stem cells.

The intracellular 2'-7'-dichlorofluoresceindiacetate (DCF-DA) levels in undifferentiated MSCs (day 0) and in cell samples collected on days 10, 20 and 30 of hepatogenic differentiation was measured. The MSCs before differentiation considered as control (100\% intensity). The results are means \pm S.D of three samples for each point. ${ }^{*} \mathrm{P}<0.05$; significant compared to control. (NS: not significant)

Figure 6.Quantitative measurement of 8-OH-dG in the mesenchymal stem cells before and after differentiation.

8-OH-dG as marker oxidative DNA damage was measured in the MSCs (day 0), and on 10, 20, and 30 days after differentiation. The results are means \pm S.D of three samples for each point. $* \mathrm{P}<0.05$; significant compared to control. (NS: not significant)

Figure 7. Expression of ATM mRNA levels measured by Q-PCR in differentiated hepatocytes and their progenitor MSCs. 
ATM expression at mRNA levels using Q-PCR technique was determined in cells recovered before differentiation (day 0 ) and cells collected on days 10, 20, and 30 of differentiation. The results are means \pm S.D of three samples for each time point. ${ }^{*} \mathrm{P}<0.05$; significant compared to control. The results of ATM expression were normalized with GAPDH-specific mRNA. (NS: not significant)

Figure 8.Immunocytochemical localization of ATM during hepatic differentiation of the MSCs.

The undifferentiated MSCs (day 0) and hepatocytes-like cells collected on days 10, 20, and 30 of differentiation were immunostained with the anti-ATM antibody (green). The nuclei were stained with DAPI (4', 6-diamidino-2-phenylindole) (blue).

Figure 9.Immunocytochemical localization of phosphorylated ATM during hepatic differentiation of the MSCs.

The undifferentiated MSCs (day 0) and hepatocytes-like cells collected on days 10, 20, and 30 of differentiation were immunostained with the anti-phosphorylated ATM (phospho S1981) antibody (green). The nuclei were stained with DAPI (4', 6-diamidino-2-phenylindole) (blue). 
Table 1. Sequence of primers used in RT-PCR for the expression of liver marker genes.

\begin{tabular}{|c|c|c|c|c|}
\hline Genes & Primer sequence & $\begin{array}{c}\text { Annealing } \\
\text { temperature, }{ }^{\circ} \text { C }\end{array}$ & produced size, bp & Genebank code \\
\hline $\begin{array}{c}\text { Cytokeratin 18 } \\
\text { (CK-18) }\end{array}$ & $\begin{array}{c}\text { F: TGG CGA GGA CTT TAA TCT TGG } \\
\text { R: CTC AGA ACT TTG GTG TCA TTG G }\end{array}$ & 55 & 128 & NM_199187 \\
\hline $\begin{array}{c}\text { Albumin (ALB) } \\
\text { R: CTTTCAAAGCATGGGCAGTAGC }\end{array}$ & 57 & 142 & NM_000477 \\
\hline $\begin{array}{c}\text { Glyceraldehyde- } \\
\text { 3-phosphate } \\
\text { dehydrogenase } \\
\text { (GAPDH) }\end{array}$ & $\begin{array}{c}\text { F: AGAGTCATCATTGGCACATTCAAGCA } \\
\text { R: AGGTTCTTCACC }\end{array}$ & 60 & 163 & NM_002046 \\
$\begin{array}{c}\text { Tryptophan2,3- } \\
\text { dioxygenase } \\
\text { (TDO2) }\end{array}$ & $\begin{array}{c}\text { F: TGG GAA CTG CCT GCA TTT GG } \\
\text { R:ACA GAA TCC AAC TCC CAG AGG }\end{array}$ & 65 & 152 & NM_005651 \\
\hline
\end{tabular}




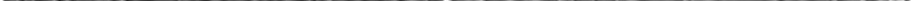


ALB

TDO2
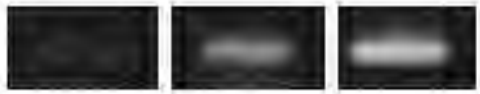

CK-18
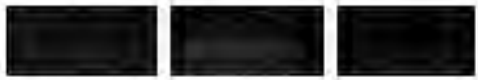

GAPDH
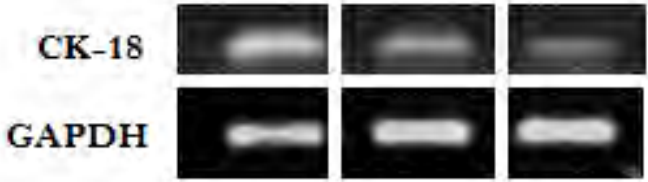


\section{A}



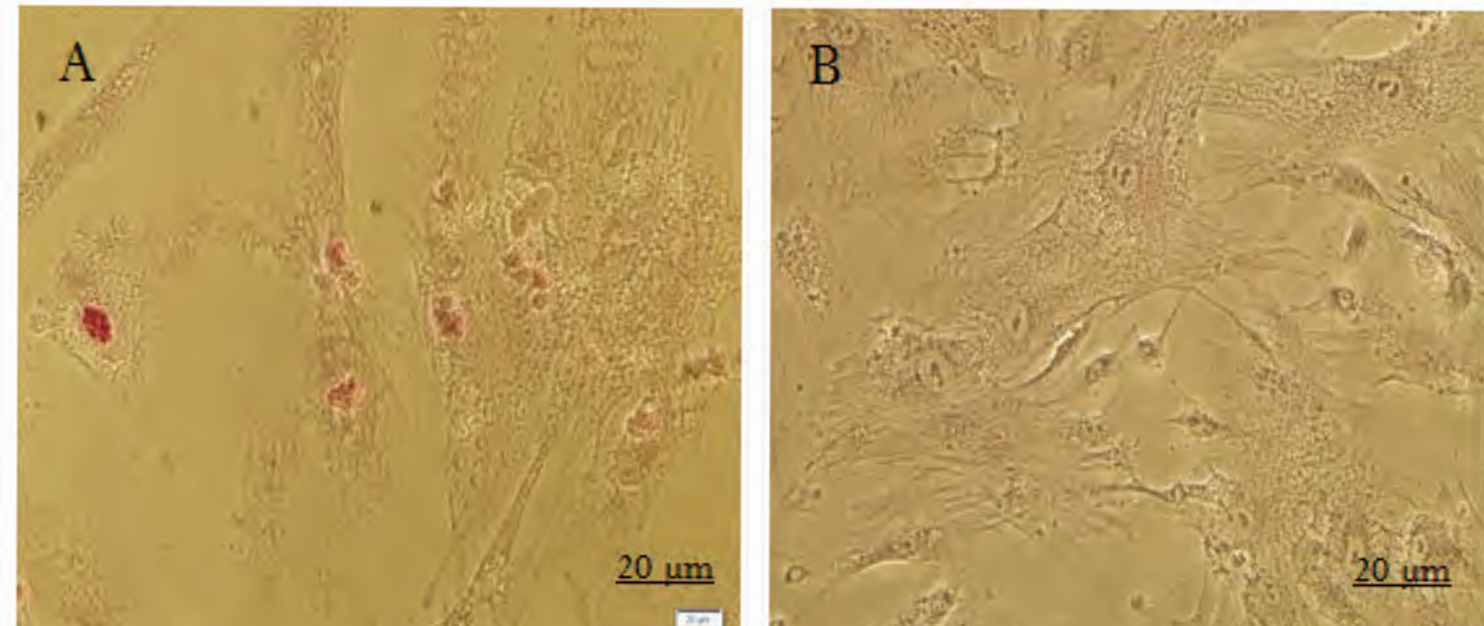
NS

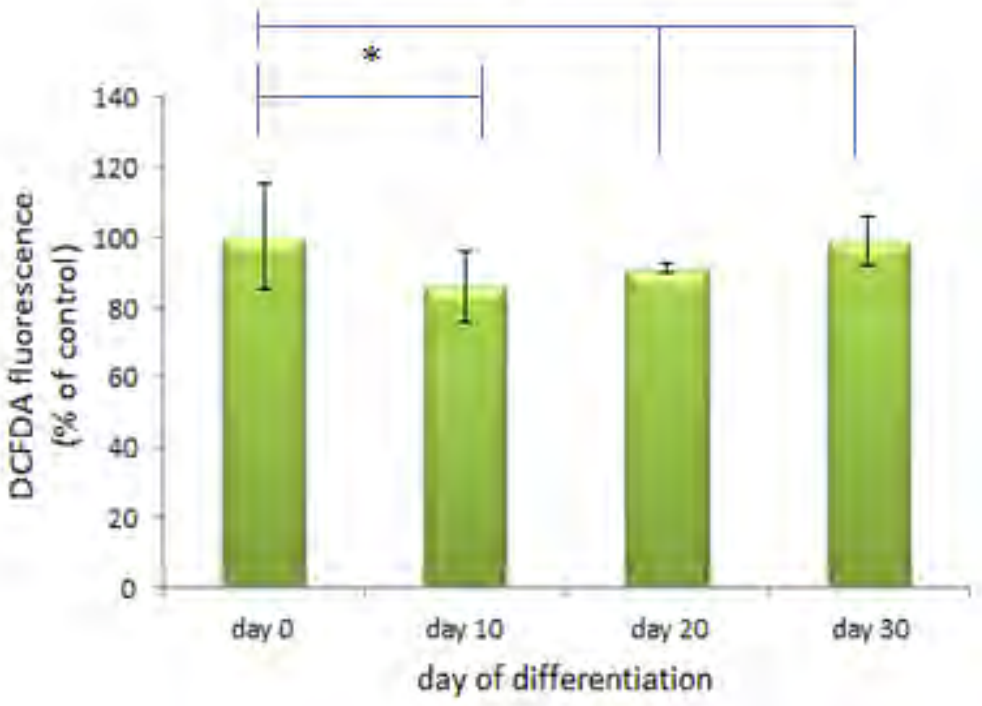




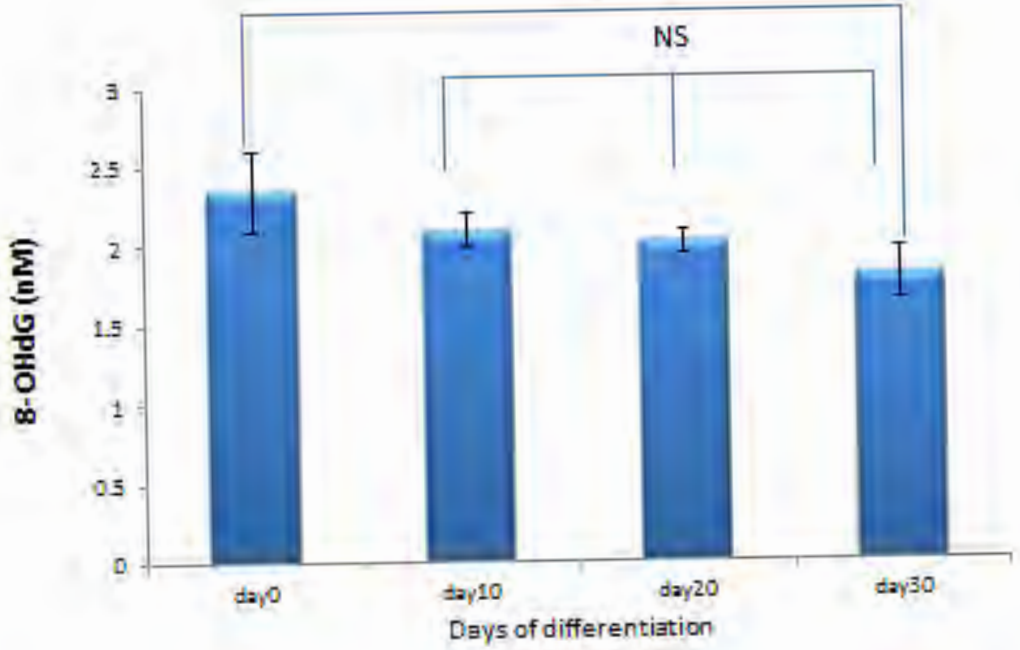


NS

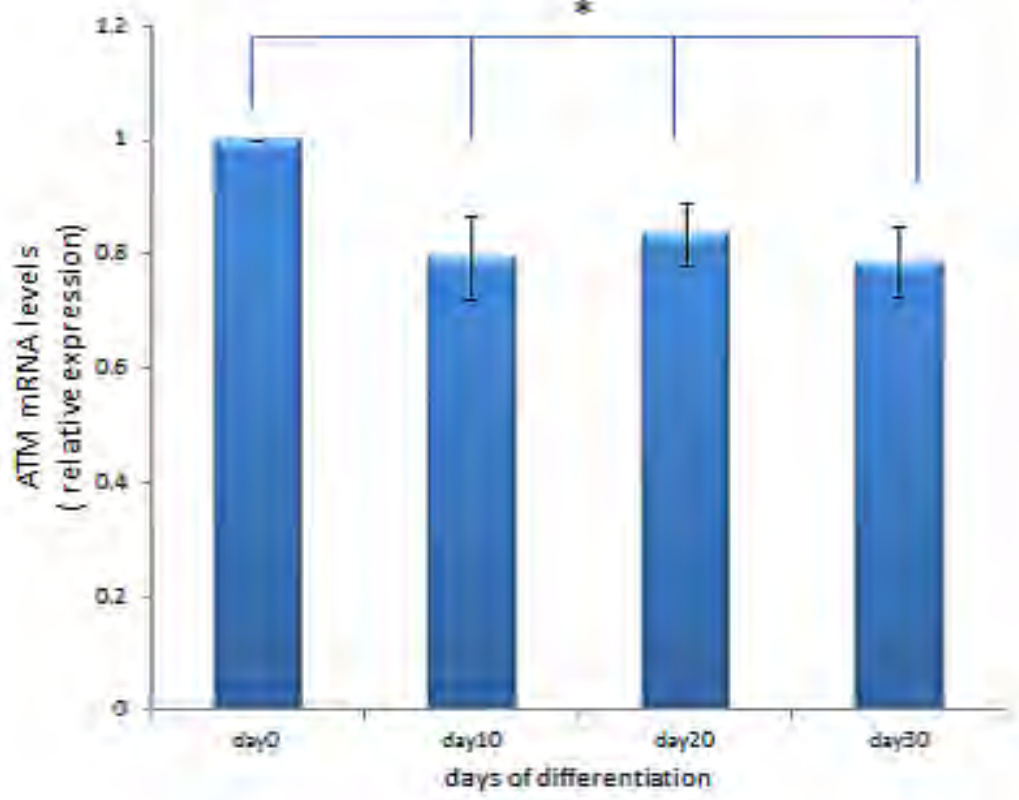



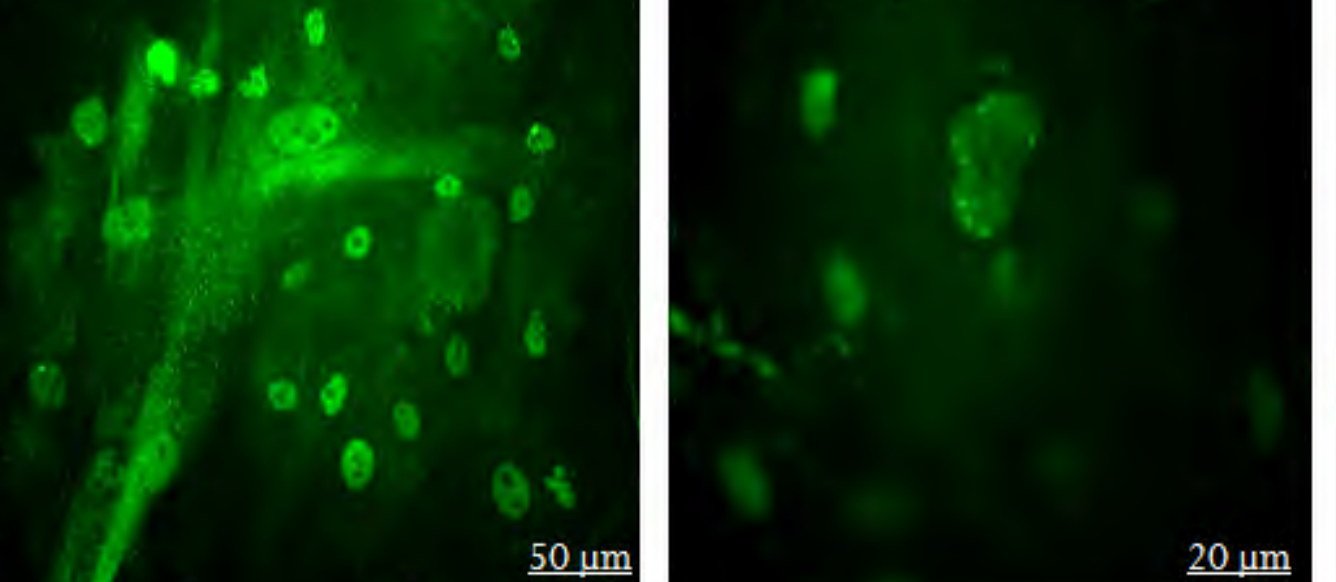

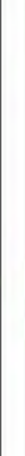
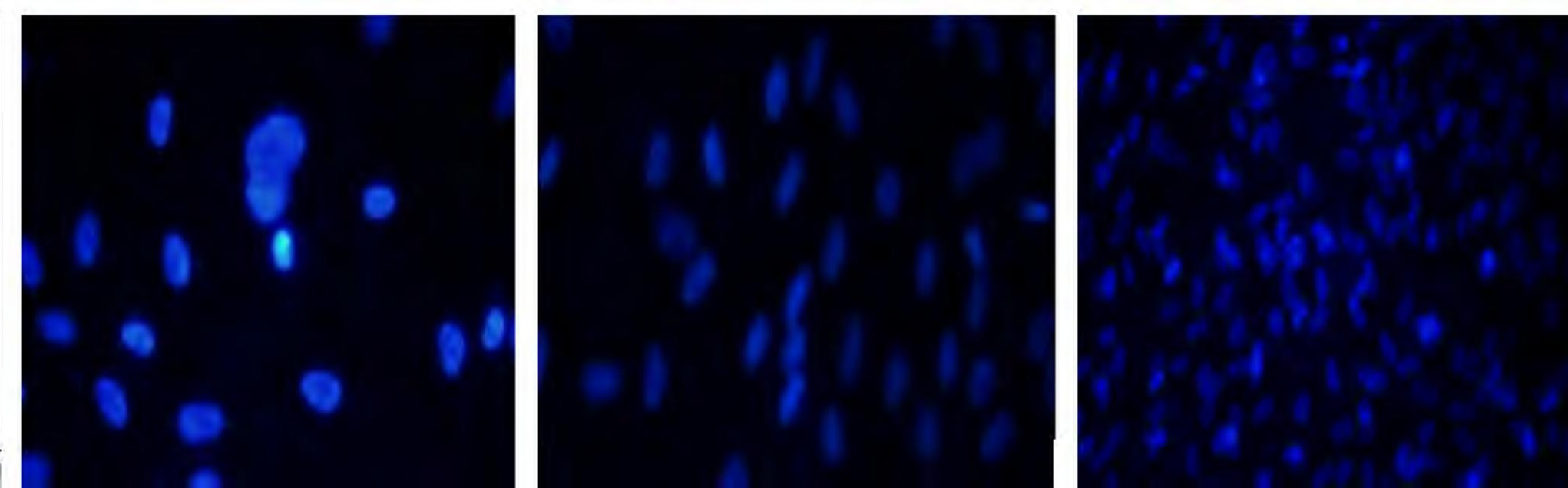